\title{
PW02-06
}

\section{THE USE OF THE FREE RECALL METHOD IN THE ANALYSIS OF VERBAL MEMORY DEFICITS IN DUAL DIAGNOSIS}

PATIENTS

K. Krysta ${ }^{1}$, A. Klasik ${ }^{2}$, M. Janas-Kozik ${ }^{1}$, J. Przybylo ${ }^{1}$, I. Krupka-Matuszczyk ${ }^{1}$

${ }^{1}$ Psychiatry and Psychotherapy, Medical University of Silesia, Katowice, ${ }^{2}$ Department of Neuropsychology, University of Opole, Opole, Poland

In schizophrenia there are specific cognitive deficits including verbal memory deficits. The objective of this study was to examine short-term verbal memory differences between subjects with and without a dual diagnosis.

A group of 80 patients with a diagnose of schizophrenia were examined. 40 of them never used illicit drugs, the other 40 also received a diagnose of addiction to psychoactive substances. The Free Recall Method was used to compare two examined groups. The results of addicted and not addicted schizophrenic patients were analyzed in all trials of the 5 stages of the examination with the use of Free Recall Method. Persons suffering from schizophrenia can usually repeat much fewer words in all five stages of the examination with Free Recall Method. This deficiency of short-term memory in this group of subjects is present from the very beginning of information coding.

As it was expected a deficiency in short-term verbal memory was found in all examined patients. However when comparing both groups no statistical differences concerning verbal memory deficits were found between the group of patients suffering from schizophrenia addicted and not addicted to psychoactive substances.

The fact that no significant differences concerning short-term verbal memory between two examined groups were found, which is consistent with some of the studies found in the literature, may be a good predictor for this group in terms of their possible chances of successful rehabilitation. 